\title{
El Comité de auditoría como herramienta del gobierno corporativo en el sector financiero
}

The audit committee as a tool for corporate governance in the financial sector Jaime Guevara Sanabria iD Universidad de Antioquia, Medellín, Colombia. Manuela Arbeláez Bermúdez iD Universidad de Antioquia, Medellín, Colombia. Luis Felipe Amaya Agudelo Universidad de Antioquia, Medellín, Colombia. Luis Fernando Yepes Gallego (ID Universidad de Antioquia, Medellín, Colombia.

\section{Autores de Correspondencia}

jalberto.guevara@udea.edu.co manuela.arbelaezb@udea.edu.co

Ifelipe.amaya@udea.edu.co luis_13940@hotmail.com

Recibido: 13-09-19

Aceptado: 06-11-19

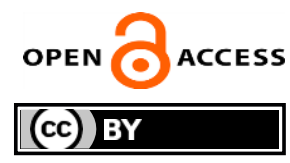

Copyright (c) 2019

Desarrollo Gerencial

\section{Resumen}

Objetivo: analizar las características de los comités de auditoría de las empresas que pertenecen al sector financiero en Colombia y están registradas en el mercado público de valores de Colombia. Método: investigación de carácter cualitativa, mediante análisis documental realizado a los reportes integrados de las entidades estudiadas. Resultados: Se identificó que los miembros de los comités de auditoría tienen deficiencias en el cumplimiento de los parámetros internacionales de independencia. En cuanto a la experiencia y formación profesional se observó adecuados parámetros de cumplimiento con una diversidad de profesiones de las ramas de las ciencias económicas que son las que en principio deberían componer los comités. Llama la atención en la dificultad para conseguir reportes de información corporativa, a pesar que el sector financiero en Colombia es altamente regulado. Discusiones: Los comités de auditoría deben garantizar la transparencia y calidad en la información financiera brindada a los diferentes usuarios, la ausencia de independencia, ponen en duda la eficacia de los comités. Conclusiones: La debilidad encontrada en la independencia podría acarrear perjuicios en el desempeño de los comités de auditoría desvirtuando así su creación y funcionamiento.

Palabras clave: Comité de auditoría, independencia, gobierno corporativo, sector financiero.

Clasificación JEL: M42

\section{Abstract}

Objective: this paper aims to analyze the audit committees of financial companies registere in the Colombian public stock market. Method: a qualitative research, through a documentary analysis based on the companies' integrated reports. Results: members of the audit committees have deficiencies related to the compliance of the international parameters of independence. In relation with experience and professional training, good compliance parameters, a variety of professions from the branches of economic sciences that are the principle of the committees were observed. Finally we took into account the difficulty to get corporate information reports needed to conduct this research, although the financial sector in Colombia is highly regulated. Discussions: Audit committees must guarantee transparency and quality in the data provided to different users, the absence of independence, they question the effectiveness of the committees. Conclusions: the weakness found in relation with independence could cause damage to the performance of the audit committees, undermining the creation of such committees.

Keywords: Audit Committee, independence, corporate governance, financial Sector.

JEL Classification: M42

Como citar este artículo (Apa):

Guevara, A., Arbeláez, M., Amaya, L. \& Yepes. L. (2019). El Comité de auditoría como herramienta del gobierno corporativo en el sector financiero. Desarrollo Gerencial, 11(2), 1-18. DOI: https://doi.org/10.17081/dege.11.2.3625 


\section{Introducción}

A nivel gerencial una de las evoluciones que se han generado para controlar los diferentes riesgos que se presentan en cuanto al reporte de los estados financieros de las empresas es la creación de un Comité de Auditoría (en adelante CA), que según Redhwan, Almahdi y Bakr, (2018), se convierte es una herramienta de defensa y autorregulación del gobierno corporativo que ayuda a supervisar de manera transparente y eficiente toda la información financiera que es llevada a cabo en todos los procesos de la organización.

De esta manera, ante la ausencia de investigaciones previas en Colombia, que involucren la eficacia, funcionamiento y composición de los CA, se desarrolló este estudio el cual tuvo por objetivo realizar la caracterización y análisis de la composición de los $\mathrm{CA}$, para lo cual, se consideró la variable de independencia como la más importante ya que determina realmente la efectividad de los CA (Rozas, 2014).

Para el desarrollo de este trabajo se tomó como base algunas referencias internacionales sobre la composición, funcionamiento y definición de las principales variables de los CA. Ante esto, se presenta primeramente el estado del arte sobre la importancia y necesidades para crear los CA, posteriormente se describe la evolución y condiciones actuales de los CA en Colombia seguido a ello se presenta desde el punto de vista teórico las principales variables que deben tener los miembros del CA y finalmente se exponen los resultados para los CA de las empresas del sector financiero en Colombia.

\section{Fundamentación teórica}

\section{La importancia de los comités de auditoría en las empresas.}

El CA ha sido una herramienta se ha venido fortaleciendo en las compañías debido a que facilita la supervisión de los procesos contables y la información financiera que presentan las organizaciones a sus usuarios, con el objetivo de brindarle a los mismos información de alta calidad y que cumplan con el principio de transparencia (Meigs \& Johnson, 1990); este comité es establecido por los miembros de la Junta Directiva; para el caso Colombiano el miembro independiente de la Junta Directiva debe estar presente en el CA de forma obligatoria, para las empresas que están bajo la inspección y vigilancia de la Superintendencia Financiera de Colombia, al igual que las que emiten instrumentos financieros negociables, esta última obligación se expande a la mayoría de países internacionales, con el objetivo de proporcionar cada vez información más confiable y transparente a los accionistas y usuarios en general.

De esta manera en este estudio se trata el tema de CA y su evolución en empresas a nivel mundial y en empresas colombianas, con el fin de crear un paralelo que proporcione información sobre la importancia que se le da a los CA, incluyendo su regulación y evolución. 
La necesidad de crear el comité de auditoría.

La necesidad de crear el CA se genera con los grandes escándalos financieros ocasionados a partir de los años 70, es así como el regulador de Estados Unidos, fomentó en las compañías la implementación de los CA con el objetivo de salvaguardar la transparencia de la información financiera. Su principal función es garantizar la fiabilidad y calidad de la información contable que difunden las compañías (Biedman, 2012). Asimismo, los CA se identifican los CA como aquella herramienta encargada de proteger los derechos de los accionistas, es decir, como una respuesta a teoría de la agencia, y de los usuarios de la información, en relación con la calidad y la transparencia de la información financiera (García \& Fernández, 2007).

Para la mayoría de los autores consideren que el CA juega un papel clave en la transparencia de la información financiera, hay otros que consideran falencias en el papel del CA, entre algunos se encuentra la percepción de estas prácticas corporativas como innecesarias por parte del mercado, ya que no ven que este atribuya una actuación diferente a las funciones que brinda el auditor externo (García \& Fernández Méndez, 2007).

Por otro lado, la ley estadounidense Frente a los fraudes contables (Ley Sarbanes-Oxley, 2002), citado en Rozas (2014), define el Comité de Auditoria como una junta o comité establecido por la Junta Directiva de una compañía emisora, que tiene por objetivo supervisar los procedimientos del sistema de información financiera incluyendo el proceso de auditoría tanto externa como interna.

De esta forma, se puede afirmar que la implementación de esta ley y los CA generan unos costos bastante altos y sus accionistas no consideran que esta implementación sea una buena inversión, esto a su vez contradice que el beneficio de tal implementación es efectivamente percibido por las compañías, aunque gran parte de las compañías consideran que el costo de la aplicación es mayor a los beneficios obtenidos (Chiple, 2005). Este argumento es debatido bajo el precepto que el CA al ser establecido en la organización y por la alta gerencia es un organismo que comprende la naturaleza de los servicios establecidos y prestados por la auditoría interna de la organización, por esta razón el CA es una herramienta que facilita el ejercicio de la auditoría y puede detectar con mayor facilidad las oportunidades de mejora que hay en el negocio (Deloitte Development, 2014).

Así, debido a los reiterados casos de fraude contable que dejaron como evidencia la ineficacia que tomaban las administraciones y la gerencia para salvaguardar la información presentada se ha creado una crisis de credibilidad sobre la función que desempeñan los auditores y su aporte en la calidad de la información financiera (Biedma, Ruiz, \& Gómez, 2011).

Entre las primeras apariciones del CA se encuentra el informe elaborado por la bolsa de valores de Nueva York NYSE titulado Independent audits and auditing procedures donde se destaca que el 
Jaime Guevara Sanabria, Manuela Arbeláez Bermúdez, Luis Felipe Amaya Agudelo, Luis Fernando Yepes Gallego

nombramiento de los auditores no debe ser elegido por la gerencia sino por parte de la Junta Directiva y debe estar compuesto por miembros no ejecutivos (Biedma, 2012). Tras varias recomendaciones esta iniciativa se volvió obligatoria, especialmente para las entidades financieras y las que cotizaban en la bolsa.

De igual manera, los CA han colaborado con aspectos como lo son la teoría de la agencia y la teoría de las señales, ya que evitan el riesgo moral dentro de las compañías al igual que los conflictos de interés entre los diversos agentes, además protege a los usuarios de la información (Monterrey \& Sánchez, 2008). En este sentido, el CA genera un puente de comunicación entre la administración y los auditores externos garantizando total transparencia de la información proporcionada entre las partes, teniendo en cuenta que su información no se puede ver afectada por los intereses particulares de las directivas (Lima, 2004).

\section{Comité de auditoría en Colombia, una herramienta efectiva para la vigilancia y el control.}

Las fallas del gobierno corporativo ocurridas en los mercados más desarrollados, también se han presentado a nivel local, como por ejemplo, la crisis del sector cooperativo a finales de los años noventa, que dejó fallas en el sistema de gobierno de las entidades financieras. Ante esta situación, el sector empresarial colombiano ha venido evolucionando en las prácticas de buen gobierno, con lo cual se han generado avances con respecto a la regulación de los CA.

La primera aparición de una norma que involucró un organismo que supervisara y vigilara la eficacia del control se realizó en el año 1998 por medio de la Circular Externa 052 de 1998 emitida por la Superintendencia Financiera de Colombia. Posterior a ello, aparece la ley que reglamenta las actividades de manejo, aprovechamiento e inversión de recursos captados del público mediante la bolsa de valores (Ley 964, 2005), con la cual se realiza una modificación al mercado público de valores.

En el artículo 45 de esta ley (ley 964, 20015), se establece la obligación que los emisores de valor constituyan un CA, el cual debe estar integrado por lo menos por tres miembros de la junta directiva incluyendo todos los independientes; además otorga funciones parecidas a la de la normatividad americana e indica que se debe reunir al menos cuatro veces en el año.

Años más tarde se actualizó y se presentó el código de mejores prácticas corporativas El Código País por medio de la Circular Externa 028 de 2014 y la Circular Básica Jurídica Circular Externa 029 de 2014 expedida por la Superintendencia Financiera de Colombia, en la cual se mencionan aspectos específicos como el mercado es quien evalúa y califica a cada emisor según la información emitida, además sugiere medidas para mejorar la práctica de los CA en las empresas (Deloitte Development, 2015).

En este punto es importante mencionar que, con base en las encuestas de Código País, se estudió los $\mathrm{CA}$, cuyos resultados indican que a Colombia le falta mucho trabajo para alcanzar niveles adecuados de gobiernos corporativos. (Lagos \& Vecino, 2013). Sin embargo, desde el punto de vista teórico el CA mejora el cumplimento legal, de control interno, de las empresas, incluyendo los riesgos como Riesgo operativo 
Jaime Guevara Sanabria, Manuela Arbeláez Bermúdez, Luis Felipe Amaya Agudelo, Luis Fernando Yepes Gallego

(SARL); Riesgo de Crédito (SARC); Riesgo operativo (SARO) y Riesgo de Lavado de Activos y Financiación del Terrorismo (SARLAFT) (Deloitte, 2015). Finalmente, ante el gran cambio en los recursos de las empresas especialmente por factores externos como la globalización, es necesario que el sistema de información contable involucre estos cambios, para tenerlos en cuenta al momento de tomar decisiones apropiadas sobre las firmas (Gómez, Aristizábal \& Fuentes, 2017; Annherys, Jaiham \& García, 2015) la correcta incorporación de los cambios, debe ser una de las funciones del CA.

\section{Principales variables de un comité de auditoría exitoso.}

En la conformación de los CA hay características de composición fundamentales, debido a que pueden afectar de manera directa la eficacia y el cumplimiento de los objetivos de este (Abbott \& Parker, 2000). Para el presente trabajo se evaluaron características que los investigadores consideraron las más relevantes, tales como independencia, experiencia, género y estudios; de igual manera el $\mathrm{CA}$, usualmente tiene entre su reglamentación mecanismos de entrenamiento para los miembros del comité, adicionalmente es importante que se tengan indicadores de autoevaluación para poder analizar la eficacia del CA y reforzar así los puntos débiles; como se mencionó anteriormente depende de la gerencia y la administración controlar la composición de los CA a fin de garantizar la eficiencia de los objetivos del mismo y generar confianza y garantiza en los intereses de los Stakeholders.

\section{Independencia: variable primordial en la toma de decisiones.}

La independencia de los miembros del CA frente a las altas directivas es una variable necesaria para que el comité cumpla de manera eficiente y eficaz su principal objetivo, por lo que las empresas deben conferirle las herramientas necesarias para su desempeño, dentro de estas características una de las más importantes es la independencia (Biedma, Ruiz \& Gómez, 2011). Sin embargo, uno de los problemas, es que el CA puede ser dependiente económicamente de la misma; teniendo en cuenta esto, se espera que desde el punto de vista de un buen gobierno corporativo y desde aspectos normativos es necesario que las decisiones que tomen las empresas sobre la estructura y composición del CA vengan determinadas por el nivel de independencia económica del auditor (Biedma, Ruiz \& Gómez, 2011), es decir, entre menor sea la dependencia económica de los miembros del comité, se pretende que mayor sea la independencia de los mismos, frente a la gerencia de la compañía, generando una relación en la calidad del CA (Zhang, Zhou, \& Zhou, 2007).

De igual manera, los intereses económicos de los miembros de comité, por el temor de perder a su cliente, pueden influir en su correcta forma de actuar y en su ética, incluso hasta volverse dependiente a las decisiones y necesidades de su cliente (Rozas, 2014), esta dependencia tiene una relación negativa con las probabilidades de fraude al interior de las organizaciones (Beasley, 1996). 
En el año 1998, el presidente de la Comisión de Bolsa y Valores de Estados Unidos (por sus siglas en ingles SEC) para ese entonces, afirmó que los CA que cumplan con los requisitos de compromiso e independencia, serán los que velaran y salvaguardaran los intereses de los usuarios de la información (Magilke, Mayhew, \& Pike, 2009); como se ha mostrado anteriormente el CA es el garante de efectividad en los objetivos que tiene la organización, asimismo los mecanismos de gobierno corporativo se consideran efectivos cuando hay una alta proporción de miembros del CA que son totalmente independientes (Jamaliah, 2017), determinando así que la independencia es garante de efectividad del gobierno corporativo, y las empresas cuyo CA es independiente tienen mejores rendimientos (Hamza \& Mselmi, 2017).

Teniendo en cuenta lo anterior, cabe mencionar el caso del Reino Unido, que por la década de los años 90's tuvo varios escándalos de fraude sobre el reporte financiero, como parte de la solución se decidió la implementación de un nuevo sistema de control interno denominado Cadbury, el cual comenzó a operar en el año 1992. Entre otros, el Cadbury logró mejorar la independencia del CA con la regla de que al menos tres de cinco miembros del CA fueran totalmente independientes de la empresa, adicionalmente se evidenciaron mejoras en el sistema contable, especialmente en lo concerniente a la disminución del fraude financiero. Prácticas como las del Cadbury dieron inicio y permitieron que los CA tomaran aun mayor fuerza con la implementación de los sistemas de gobierno corporativo debido a que el mismo cumple un papel fundamental en el desarrollo del plan de control interno y gobierno corporativo (Mancilla \& Saavedra, 2015).

Para el caso colombiano, Superintendencia Financiera en su circular Externa 029 de 2014 señala que el CA debe estar compuesto por tres miembros de la Junta Directiva, incluyendo a los miembros independientes de la misma. En esta investigación se trabaja como dependencia de los miembros del CA toda situación en la que el miembro del comité o su familia, se ve influenciada por un interés secundario generalmente de tipo económico o personal.

\section{Incidencia del género en la conformación del comité de auditoría.}

La transición de una sociedad tradicionalista con altos niveles de machismo incluidos a una sociedad liberalizada ha permitido que en los últimos años las mujeres tuviesen mayor incidencia en los cargos ejecutivos de las empresas (Pucheta \& Bel, 2012), como ejemplo en España, la Ley Orgánica para la igualdad efectiva de mujeres y hombres (Ley 3, 2007), la cual tiene como objeto hacer efectivo el derecho de igualdad de trato y de oportunidades entre mujeres y hombres, en particular mediante la eliminación de la discriminación de la mujer, sea cual sea la circunstancia o condición, en cualquiera de los ámbitos de la vida y singularmente, en las esferas políticas, civiles, laborales, económicas, sociales y culturales, incorporándola además en la composición del CA. Sin embargo, en el caso colombiano aún no existe una 
Jaime Guevara Sanabria, Manuela Arbeláez Bermúdez, Luis Felipe Amaya Agudelo, Luis Fernando Yepes Gallego

norma que haga de obligatorio cumplimiento la integración de los CA con por lo menos un miembro del género femenino.

Incluso la literatura previa centrada en el gobierno corporativo, arrojó como resultados que la diversidad de género en CA puede llegar a influir, en la calidad del comité, debido a la integración de las cualidades tanto de miembros hombres como mujeres para la supervisión, administración y el control de las actividades del CA (Adams \& Ferreira, 2012). Es por esto, que por lo menos una mujer en los CA podría mejorar la calidad de la información financiera de las empresas, sin embargo, lo anterior aun no es suficiente para afirmar que con las mujeres la calidad del control interno siempre mejore, debió a la escasa investigación realizada combinando la diversidad de generó en los comités de auditoría y la calidad de la información financiera (Ittonen, Miettinen, \& Vahamaa, 2010).

Asimismo, otro indicador a favor de la diversidad de genero está orientado a que los mayores fraudes financieros han sido protagonizados por hombres, desencadenando que la relación fraude y hombres es mucho más significativa que la de fraudes y mujeres (Dinero, 2012), permite pensar que las mujeres tienen mayor independencia; por otra parte se ha logrado identificar que las mujeres pertenecientes a los CA redujeron la manipulación del devengo, lo que sugeriría un aumento en la calidad de la información financiera generando mayor confianza para todos los usuarios de la información (Qi \& Tian, 2012).

Por su parte las empresas se caracterizan por la falta de igualdad en los puestos de trabajo, lo que se considera como un maltrato profesional a causa del machismo; se afirma que la discriminación causada en los CA son debidas a la infravaloración de la eficacia de los CA con mujeres, la creencia de falta de capacidades intelectuales para llevar a cabo el plan de acción del comité, discriminación por preferencias y ventajas o desventajas que se incurren al contratar mujeres, las responsabilidades familiares y la menor remuneración frente al sexo opuesto (Pucheta \& Bel, 2012). A pesar de ello la incorporación de las mujeres en los puestos con mayores niveles jerárquicos ha sido lenta, pero en la actualidad el género femenino ha vivido una transición hasta el punto de ocupar elevados puestos con grandes responsabilidades dentro de las organizaciones que antes habían sido reservados para hombres (Carrasco \& Laffarga, 2007) por lo cual la conformación de un CA donde se cuente con por lo menos una mujer contribuirá con la igualdad de género que día a día se está alcanzando.

\section{El conocimiento y la experiencia de los miembros del comité}

Algunos de los estudios acerca de los comités de auditoría han tomado fuerza debido a los escándalos financieros que se han presentado a nivel mundial (Pucheta \& Bel, 2012), muchas de las compañías implicadas en este tipo de escándalos han tenido en su estructura organizacional bien sea por ley o por voluntad propia un CA, lo que ha llevado a serios cuestionamientos la funcionalidad del mismo, al interior de las organizaciones. 
Una de las variables que pueden están implicadas es la experiencia y perfil de los miembros del CA, para poder desempeñar a cabalidad su función en las organizaciones a las cuales pertenecen.

Dentro de la conformación de los CA se debe tener en cuenta que sus miembros deben de contar con la experiencia y conocimiento necesario de todo lo relacionado a sus funciones como miembro del CA (Pucheta \& Bel, 2012), , es muy posible que las fallas en cuanto al desarrollo de las actividades propias de los CA se estén generando porque no haya personas competentes en cuanto a la rama del conocimiento y experiencia que poseen. Las nuevas normas en el mundo dan a entender que una de las razones que afectan la eficacia de los CA es precisamente la competencia profesional (Zarza, López \& Reguera, 2018), muchas veces en las compañías se entiende como competente para ser miembro del CA una persona que tiene mucha experiencia en el sector en el que se desarrolla la empresa, si bien es importante una persona con este perfil dentro del comité, no debe ser el foco principal de selección a la hora de elegir a un miembro del comité; el perfil profesional, competencia técnica y experiencia de un miembro del comité debe enfocarse en el área de auditoría, contabilidad o finanzas (Zarza, López \& Reguera, 2018). Ejemplo de lo anterior es que, dentro de las doctrinas y conceptos del gobierno colombiano, se entiende que los miembros del CA deben ser profesionales conocedoras de su oficio (Superintendencia Financiera de Colombia, 1999).

En las doctrinas y leyes a nivel mundial también se resalta que los miembros de CA sean personas con experiencia comprobada, como es el caso de la ley SOX que manifiesta que cuando menos uno de los miembros debe tener un amplio conocimiento de ramas como la contaduría, la auditoría, administración y demás conocimientos afines como lo son las NIIF y las NIAS (Robinson \& Owens, 2009).

Se ha encontrado entonces que la competencia técnica de los miembros del CA es uno de los más importantes requerimientos al momento de conformar los mismos ya que un CA mejor preparado profesionalmente puede generar unos mejores resultados.

Para el análisis de este estudio, se trabajó como experiencia de los miembros del CA, aquellos que tenían en empresas de la rama financiera en funciones de auditoría, revisoría fiscal y demás áreas que daban cumplimiento a los objetivos del CA, y la misma no debía ser menor a 10 años. Adicionalmente, se definió como estudios los pregrados en carreras afines a las ciencias económicas como Contaduría Pública, Derecho, Administración, Ingeniería Financiera e Industrial y Economía. 


\section{Método}

Diseño.

Este estudio presente una investigación de enfoque cualitativo, con alcance exploratorio y en el cual se utilizó técnicas como el análisis documental, con el fin de determinar las características del CA de las empresas del sector financiero de Colombia.

\section{Participantes.}

La unidad de análisis corresponde a todas las empresas del sector financiero que están registradas en el mercado público de valores (BVC) siendo en total 48 de ellas, teniendo información definitiva para todas las variables de estudio en 26 empresas ya que fueron las únicas empresas del sector financiero que poseen todos los datos para el desarrollo de esta investigación.

\section{Instrumentos.}

Se utilizó como instrumento una matriz de análisis de contenido que tomó las características cualitativas de las empresas como: independencia de los miembros del CA, experiencia profesional de los integrantes del CA, título de pregrado de los miembros del CA, género, tamaño, ingresos operacionales, utilidad antes de impuestos y revisor fiscal (las 4 consultoras más grandes del mundo-Big Four, por sus siglas en inglés).

\section{Procedimiento.}

Los datos de la matriz se extrajeron de la lectura de los informes de gobierno corporativo y de gestión del año 2017, los cuales se diligenciaron de forma manual en la matriz. La fuente de los informes de gestión fueron las páginas web de las empresas de la muestra. Adiciona a ello, para el análisis de los datos de la matriz, las empresas se clasificaron en tres grupos según su tamaño (grande, mediano y pequeño), sus ingresos operacionales, la utilidad antes de impuestos y el revisor fiscal. Con esta agrupación se buscó identificar las relaciones de los CA con variables anteriormente mencionadas.

\section{Resultados}

Los resultados obtenidos se clasifican, de acuerdo con las principales variables de los CA incluyendo los respectivos análisis por tamaño de las empresas, niveles de ingresos y firma de auditoría.

\section{Independencia: variable primordial en la toma de decisiones.}

Inicialmente se identifica que de las 26 empresas de las que se encontró información sobre la composición del CA solamente el $17 \%$ de los miembros de los comités son totalmente independientes de 
Jaime Guevara Sanabria, Manuela Arbeláez Bermúdez, Luis Felipe Amaya Agudelo, Luis Fernando Yepes Gallego las compañías, al no cumplir con el criterio de independencia realizado para el presente trabajo, lo anterior es debido principalmente a que se hallaron relaciones económicas o de interés con la empresa o alguno de sus accionistas principales con los miembros independientes que hacen parte del CA o sus familiares; (Tabla 1).

Tabla 1.

Independencia de los miembros del CA

\begin{tabular}{|c|c|c|c|c|c|c|}
\hline \multirow{2}{*}{$\begin{array}{l}\text { Independencia } \\
\text { Categoría }\end{array}$} & \multicolumn{2}{|c|}{ Activos } & \multicolumn{2}{|c|}{ Ingresos Operacionales } & \multicolumn{2}{|c|}{ UAI } \\
\hline & Independientes & Dependientes & Independientes & Dependientes & Independientes & Dependientes \\
\hline Grande & $26 \%$ & $74 \%$ & $21 \%$ & $79 \%$ & $31 \%$ & $69 \%$ \\
\hline Mediana & $23 \%$ & $77 \%$ & $22 \%$ & $78 \%$ & $13 \%$ & $87 \%$ \\
\hline Pequeña & $9 \%$ & $91 \%$ & $10 \%$ & $91 \%$ & $15 \%$ & $85 \%$ \\
\hline Total & $17 \%$ & $83 \%$ & $17 \%$ & $83 \%$ & $17 \%$ & $83 \%$ \\
\hline
\end{tabular}

Se resalta que la independencia aumenta, de acuerdo al tamaño de las empresas y a sus resultados de utilidad antes de impuestos.

Por otra parte, las empresas vigiladas por la Superintendencia Financiera de Colombia y que hacen parte del mercado de valores están obligadas a tener Revisor Fiscal, a esta figura le corresponde dictaminar los estados financieros al igual que evaluar el control interno de las organizaciones. La Revisoría Fiscal cumple un papel importante a la hora de velar por la transparencia de la información financiera y evaluar el cumplimiento de la normativa y los objetivos que rigen las organizaciones; para este caso, se logró evidenciar el control total que tienen las Big-Four para el mercado de la Revisoría Fiscal de las empresas financieras del mercado público de la Bolsa de Valores, en ella solo se identificó que una de las 26 empresas evaluadas cuenta con un revisor fiscal que no pertenece al grupo de los Big-Four (Tabla 2).

Tabla 2.

Independencia/ Revisor Fiscal

\begin{tabular}{ccccr}
\hline Caracterización & \multicolumn{4}{c}{ Independencia } \\
\hline Categoría & Independientes & \multicolumn{2}{c}{ Dependientes } \\
\hline Abako's s.a. & 0 & $0 \%$ & 1 & $100 \%$ \\
\hline Deloitte & 1 & $17 \%$ & 6 & $83 \%$ \\
\hline Ey & 1 & $29 \%$ & 1 & $71 \%$ \\
\hline Kpmg & 2 & $18 \%$ & 8 & $82 \%$ \\
\hline Pwc & 1 & $14 \%$ & 5 & $86 \%$ \\
\hline Total & 5 & $17 \%$ & 21 & $83 \%$ \\
\hline & Fuente: Elaboración propia (2018). & & \\
\hline
\end{tabular}

Cabe resaltar que las firmas auditadas por la organización EY cuentan con un mayor nivel de independencia entre los miembros del CA es decir, el 29\% de los miembros del CA auditado por EY son totalmente independientes, con las otras tres empresas que conforman las Big Four, no se observan variaciones significativas en la independencia de los miembros del CA, rondando un promedio del $16 \%$. 


\section{El conocimiento y la experiencia de los miembros del comité de auditoría.}

Para la definición de esta variable se tuvieron en cuenta varios aspectos como el tiempo de trabajo en el sector financiero y sobre todo el liderazgo de los procesos relacionados con las temáticas del CA, como en temas financieros, gestión de riesgos, control interno, auditoria interna y revisoria fiscal, con un tiempo aproximado de 10 años.

En la aplicación del instrumento de investigación se observó que las empresas del sector financiero clasificadas como grandes y medianas tanto en tamaño por total de activo, utilidad antes de impuestos e ingresos operacionales el $100 \%$ de los miembros del comité tienen más de 10 años de experiencia en los cargos descritos anteriormente (Tabla 3). En cuanto a las empresas pequeñas del sector financiero, se destaca también un porcentaje alto, el $98 \%$ de miembros de CA con más de 10 años de experiencia en funciones similares.

Tabla 3.

Experiencia miembros comité de auditoría

\begin{tabular}{|c|c|c|c|c|c|c|}
\hline \multirow{2}{*}{$\begin{array}{l}\text { Experiencia } \\
\text { Categoría }\end{array}$} & \multicolumn{2}{|c|}{ Activos } & \multicolumn{2}{|c|}{ Ingresos operacionales } & \multicolumn{2}{|c|}{ UAI } \\
\hline & > 10 Años & $<10$ Años & > 10 Años & $<10$ Años & > 10 Años & $<10$ Años \\
\hline Grande & $100 \%$ & $0 \%$ & $100 \%$ & $0 \%$ & $100 \%$ & $0 \%$ \\
\hline Mediana & $100 \%$ & $0 \%$ & $100 \%$ & $0 \%$ & $100 \%$ & $0 \%$ \\
\hline Pequeña & $98 \%$ & $2 \%$ & $98 \%$ & $2 \%$ & $98 \%$ & $2 \%$ \\
\hline Total & $99 \%$ & $1 \%$ & $99 \%$ & $1 \%$ & $99 \%$ & $1 \%$ \\
\hline
\end{tabular}

Asimismo, en este estudio el conocimiento se relaciona con estudios o desarrollos afines a las ciencias contables, administrativas y económicas como Contaduría Pública, Derecho administrativo, Administración, Ingeniería Financiera e Industrial y Economía.

Teniendo en cuenta que las anteriores profesiones desarrollan en sus contenidos de formación ítems claves en las funciones del CA como Normas Internacionales de Información Financiera, Normas Internacionales de Aseguramiento, Sistemas de Control Interno, metodologías de identificación de Riesgos, temas relacionados con auditoría entre otros, se encontró como resultado, que en el caso de las empresas del sector financiero en Colombia el 78\% de los miembros del CA tienen conocimientos en carreras afines. También se destaca que las medianas empresas cuentan con el $86 \%$ de los miembros con carreras afines. (Tabla 4). Se resalta que la participación de los Contadores Públicos en los CA es prácticamente nula, ya que solo se encontró un Contador Público como miembro de CA (Tabla 4). 
Tabla 4.

Profesión de los miembros del CA

\begin{tabular}{cccccccc}
\hline Profesión & \multicolumn{2}{c}{ Activos } & \multicolumn{2}{c}{ Ingresos Operacionales } & UAI \\
\hline Categoría & Carreras afines & Carreras diferentes & Carreras afines & Carreras diferentes & Carreras afines & Carreras diferentes \\
\hline Grande & $74 \%$ & $26 \%$ & $79 \%$ & $21 \%$ & $69 \%$ & $31 \%$ \\
\hline Mediana & $84 \%$ & $16 \%$ & $70 \%$ & $30 \%$ & $82 \%$ & $18 \%$ \\
\hline Pequeña & $76 \%$ & $24 \%$ & $85 \%$ & $15 \%$ & $79 \%$ & $21 \%$ \\
\hline Total & $78 \%$ & $22 \%$ & $78 \%$ & $22 \%$ & $78 \%$ & $22 \%$ \\
\hline
\end{tabular}

Fuente: Elaboración propia (2018).

Los anteriores resultados se deben analizar junto con la experiencia y ambos variables tanto participación de las carreras afines como la experiencia de más de 10 años la consideramos alta.

\section{Incidencia del género en la conformación del comité de auditoría.}

Actualmente en Colombia el tema del género ha cobrado gran importancia, un ejemplo es el documento emitido por la Alta consejería Presidencial para la equidad de la Mujer (2012) donde se establecen una serie de políticas de estado de igualdad de género; asimismo, dentro de los Objetivos de Desarrollo Sostenible (ODS) establecidos por el Programa de las Naciones Unidas para el Desarrollo (2015), en su objetivo $\mathrm{N}^{\circ} 5$ incluye la igualdad de género como un efecto multiplicador que ayuda a promover el crecimiento a nivel mundial, por lo cual cada día se espera que sean más las mujeres que ocupen un protagonismo dentro de las organizaciones. Para este caso del comité de auditoría en particular se tienen los siguientes datos:

Tabla 5.

Género de los miembros del CA

\begin{tabular}{|c|c|c|c|c|c|c|}
\hline \multirow{2}{*}{$\begin{array}{c}\text { Género } \\
\text { Categoría }\end{array}$} & \multicolumn{2}{|c|}{ Activos } & \multicolumn{2}{|c|}{ Ingresos operacionales } & \multicolumn{2}{|c|}{ UAI } \\
\hline & Hombre & Mujer & Hombre & Mujer & Hombre & Mujer \\
\hline Grande & $90 \%$ & $10 \%$ & $92 \%$ & $8 \%$ & $100 \%$ & $0 \%$ \\
\hline Mediana & $76 \%$ & $24 \%$ & $78 \%$ & $22 \%$ & $68 \%$ & $32 \%$ \\
\hline Pequeña & $81 \%$ & $19 \%$ & $78 \%$ & $22 \%$ & $83 \%$ & $17 \%$ \\
\hline Total & $82 \%$ & $18 \%$ & $82 \%$ & $18 \%$ & $82 \%$ & $18 \%$ \\
\hline
\end{tabular}

En la Tabla 5, se presenta la clasificación según la utilidad antes de impuestos, Se puede observar que en las grandes empresas la variable incidencia de género, arroja que ninguna mujer es miembro del CA. Caso contrario es en las medianas empresas donde se observa una mayor participación del género femenino con un $22 \%$ en la clasificación por tamaño y un $32 \%$ en la clasificación por utilidad antes de impuestos, lo que es considerado positivo para la transición igualitaria, sin embargo, en términos generales aún falta mucho para que las mujeres tengan mayor relevancia en los cargos ejecutivos de las empresas como por ejemplo en los CA. 
Por otra parte, los resultados muestran que las empresas del sector financiero en Colombia que eligen revisor fiscal a DELOITE tienen un 43\% de mujeres integrando sus CA, todo lo contrario, sucede con la firma KPMG donde solo un 6\% de los miembros del CA de las empresas que audita son mujeres.

\section{Discusiones}

Consideramos que una de las variables más importantes en los CA es la independencia, sin embargo, con los resultados obtenidos se da a entender que hay empresas que no gozan de miembros totalmente imparciales, y esto puede llegar a afectar el debido funcionamiento del comité y afectar los controles de fraude. Tal como lo mencionan (Biedma, Ruiz \& Gómez, 2011) en el presente estudio se presenta un conflicto al tratar de medir e identificar la independencia de los miembros del CA con la gerencia, debido a que se evidencia la poca relevancia que tienen las empresas para indagar y utilizar medios para garantizar la independencia de los miembros del CA. Esta situación se acentúa con la clasificación de empresas pequeñas las cuales muestran un resultado de independencia de tan solo el $9 \%$, logrando identificarse que entre mayor es la independencia, mayor es el tamaño de la empresa en cuanto a sus activos y sus ingresos operacionales.

Se observa también que las empresas más grandes tienen una mayor responsabilidad corporativa frente a sus accionistas y usuarios de la información, por lo que es común que estas empresas tengan en cuenta la independencia en mayor proporción que las empresas con un tamaño inferior de los miembros independientes del CA. Estos resultados están en la misma línea de lo investigado por Hamza y Mselmi (2017) que concluyen que las empresas con CA más independientes tienen unos mayores rendimientos. Adicionalmente leyes como Sarbannes-Oxley y las regulaciones de la Superintendencia Financiera afirman que la mayoría de los miembros de los comités deben ser independientes (por lo menos tres de sus cinco miembros), es decir, el $60 \%$ de los miembros de un comité deben de ser totalmente independientes de la gerencia, situación que no se estaría presentando en la caracterización de los CA de las empresas del sector financiero.

Así mismo la experiencia profesional según Zarza, López y Reguera (2018), es clave para el funcionamiento de los CA lo que de alguna manera los hace idóneos por la variable de experiencia, en el trabajo realizado se encuentra una buena experiencia de los miembros de CA, este resultado está de acuerdo con Pucheta y Bell (2012), quienes son enfáticos qne que los miembreos del CA deben tener suficiente experiencia para el cumplimiento de sus funciones.

Por otra parte, cabe resaltar que en cuanto al conocimiento, aunque se encuentran como carreras afines, solo encontramos un Contador Público como miembro del CA, lo anterior dejan abierto algunos interrogantes ¿la preparación o el perfil del Contador Público no tiene un perfil directivo? o quizá ¿a los Contadores Públicos no les interesa pertenecer a este tipo de comités en las organizaciones?, el punto es 
Jaime Guevara Sanabria, Manuela Arbeláez Bermúdez, Luis Felipe Amaya Agudelo, Luis Fernando Yepes Gallego

que se considera que el contador público es el profesional que posee las bases más sólidas en cuanto a la auditoría, NIIF y NIA's, sin embargo, en la práctica no está representando una participación significativa en los CA de las empresas dejando abiertas las posibilidades a otros profesionales a incursionar en estos órganos de control y de alguna manera ir dejando de lado funciones que son muy propias de la profesión. Estos resultados están acordes con una de las principales críticas a nivel mundial de los CA que es la falta de conocimientos en auditoría financiera, por parte de sus miembros.

Por otro lado, los resultados obtenidos en la composición de género permiten deducir que, aunque estamos en la transición hacia una inclusión de la mujer en cargos de alta jerarquía como por ejemplo ser miembro de los CA, aún falta mucho para para llegar a una igualdad del género, pues entre más grande se considere la empresa, menos mujeres hacen parte de sus CA.

Desde el punto de vista teórico, esta baja composición de mujeres en los comités puede incidir en una mayor cantidad de fraudes de acuerdo con el trabajo de Qi \& Tian (2012), que indica que entre mas sea el número de mujers en los CA menos correacion de fraude presentan las respectivas empresas.

\section{Conclusiones}

A partir del estudio realizado se considera que aunque en Colombia existe normatividad que regula los $\mathrm{CA}$, aún falta gestión por parte de las compañías para equiparar a los parámetros internacionales, incluyendo el Código de Ética de la IFAC, especialmente en los relacionado con la variable de independencia, esta situación podría afectar el correcto desarrollo y la toma de decisiones por parte de los miembros del comité en la ejecución de sus funciones perjudicando la esencia misma del comité y desvirtuándola de sus objetivos principales.

A pesar de la normatividad vigente y del desarrollo del ODS No. 5 se evidencia desigualdad en la equidad de género en los miembros del CA. Aún falta avanzar en el camino para seguir cerrando las brechas de desigualdad.

Por otro lado, se logró evidenciar que los miembros del CA son personas altamente capacitadas con experiencia financiera, sin embargo, no obstante el profesional de las Ciencias Contables no está participando en los diferentes CA, profesión que se considera la más idónea por el desarrollo temas de auditoria como Normas de Aseguramiento NIIF y riesgos.

Finalmente, este trabajo abre la apertura a futuros trabajo de investigación incluyendo metodologías cuantitativas, que puedan ser útiles para medir la eficacia y la calidad de los mismos y compararlos con variables como desempeño pagos de dividendos, valoración en el mercado, minimización de riesgo (SARL o SARC) e inclusive con variables como disminución del fraude. 


\section{Referencias}

Abbott, L. \& Parker, S. (2000). Auditor Selection and Audit Committee Characteristics. Journal of Practice \& Theory, 19(2), 47-66. DOI: https://doi.org/10.2308/aud.2000.19.2.47

Adams, R., \& Ferreira, D. (2012). Regulatory pressure and bank directors incentives to attend board meeting. International Review of Finance. 12(1), 227-248. DOI: https://doi.org/10.1111/j.14682443.2012.01149.x

Alta consejería Presidencial para la equidad de la Mujer. (2012). Lineamiento de la política pública nacional de equidad de género para las mujeres. Bogotá: Colombia. Recuperado de http://www.equidadmujer.gov.co/Documents/Lineamientos-politica-publica-equidad-de-genero.pdf

Annherys, P., Jaiham, H. \& García, J. (2015). Toma de decisiones: reto para crear ventajas competitivas en las distribuidoras de alimentos gourmet. Desarrollo Gerencial, 72 ), 100-118. DOI: https://doi.org/10.17081/dege.7.2.1183

Beasley, M. (1996). An Empirical Analysis of the Relation between the Board of Director Composition and Financial Statement Fraud. American Accounting Association, 71(4), 443-465. Recuperado de: https://www.jstor.org/stable/248566?seq=1\#page_scan_tab_contents

Biedma-López, E. (2012). El comité de auditoría como salvaguarda de la independencia del auditor. Evidencias empíricas para el caso español(Tesis Doctoral, Universidad Pablo de Olavide). Recuperado de: http://www.icac.meh.es/Documentos/PUBLICACIONES/275.pdf

Biedma-López, E., Ruiz-Barbadillo, E. \& Gómez-Aguilar, N. (2011). ¿Cómo actúan las empresas frente a la dependencia económica del auditor?: el papel del comité de auditoría. Revista de contabilidad, 14(1), 87-119. DOI: https://doi.org/10.1016/S1138-4891(11)70023-1

Carrasco, A. \& Laffarga, J. (2007). La diversidad de género en el Código Unificado español y la práctica empresarial. Pecunia, (4), 1-25. Recuperado de http://hdl.handle.net/11441/16265

Chiple, B. (2005). Implementación y Estado de Sarbanes Oxley en las compañías chilenas. (Tesis de pregrado, Universidad de chile). Recuperado de: http://www.tesis.uchile.cl/tesis/uchile/2005/chiple_b/sources/chiple_b.pdf

Congreso de Colombia. (8 de julio de 2005). Ley que reglamenta las actividades de manejo, aprovechamiento e inversión de recursos captados mediante valores. [Ley 945, 2005]. DO: 45.963. 
Congreso de los Estados Unidos. (30 de julio de 2002). Ley Frente a los fraudes contables. [Ley SarbanesOxley de 2002]. Recuperado de http://interamerican-usa.com/articulos/Leyes/Ley-Sar-Oxley.htm

Corte General de España. (22 de marzo de 2007). Ley Orgánica para la igualdad eféctiva de mujeres y hombres [Ley 3, 2007). Recuperado de https://www.boe.es/buscar/act.php?id=BOE-A-2007-6115

Deloitte Development LLC. (2015). Comité de Auditoría Órgano de apoyo a la Junta Directiva Recuperado de https://www2.deloitte.com/co/es/pages/risk/articles/comite-de-auditoria-colombia.html

Deloitte Development LLC. (Octubre, 2014). Auditoría Interna: Moviéndose más allá del cumplimiento. Recuperado de Deloitte. Recuperado de: https://www2.deloitte.com/ni/es/pages/audit/topics/audit-committee-brief/auditoria-interna-masalla-del-cumplimiento.html

García-Cabal, M. \& Fernández-Méndez, C. (2007). Efectos económicos de la constitución de los comités de auditoría. Revista de Contabilidad-Spanish Accounting Review, 10(2), 103-124. Recuperado de https://revistas.um.es/rcsar/article/view/388891

Gómez-Cano, C., Aristizábal-Valbuena, C. \& Fuentes-Gómez, D. (2017). Importancia de la información financiera para el ejercicio de la gerencia. Desarrollo Gerencial, 9(2), 88-101. DOI: https://doi.org/10.17081/dege.9.2.2977

Hamza, T. \& Mselmi, N. (2017). Corporate Governance and Equity Prices: The Effect of Board of Directors and Audit Committee Independence. Management International, 21(2), 152-164. DOI: https://doi.org/10.7202/1052694ar

Ittonen, K., Miettinen, J. \& Vahamaa, S. (2010). Does female representation on audit committees affect audit fees? Quarterly Journal of Finance and Accounting, 49(3/4), 113-139. Recuperado de https://www.jstor.org/stable/23074633

Jamaliah, A. (2017). Audit committee independence and a contracting perspective on good will impairment: singaporean evidence. Business: Theory \& Practice, 18(1) 128-135.DOI: https://doi.org/10.3846/btp.2017.013

Lagos, D. \& Vecino, C. (2013). Medición de la aplicación de prácticas de gobierno corporativo en Colombia. Tendencias, 12(2), 230-253. Recuperado de http://revistas.udenar.edu.co/index.php/rtend/article/view/535 
Lima, R. (2004). Gobierno Corporativo: Una comparación entre empresas de México, Estados Unidos y Alemania. (Tesis de pregrado, Universidad de las Américas Puebla). Recuperado de http://catarina.udlap.mx/u_dl_a/tales/documentos/lad/lima_t_ra/

Los mayores fraudes financieros. (2012). Dinero. Recuperado de https://www.dinero.com/inversionistas/articulo/los-mayores-fraudes-financieros/155137

Magilke, M., Mayhew, B. \& Pike, J. (2009). Are independent audit committee members objective? Experimental evidence. The Accounting Review, 84(6), 1959-1981.DOI: https://doi.org/10.2308/accr.2009.84.6.1959

Mancilla, M. \& Saavedra, M. (2015). El gobierno corporativo y el comité de auditoría en el marco de la responsabilidad social empresarial. Contaduría y administración.60(2), 486-506. DOI: http://dx.doi.org/10.1016/S0186-1042(15)30011-5

Meigs, W. \& Johnson, C. (1990). Contabilidad, la base para las decisiones comerciales. Bogotá: McGrawHill.

Monterrey, J. \& Sánchez, A. (2008). Gobierno corporativo y calidad de la información contable. Revista de contabilidad: Spanish accounting Review, 11(1), 65-98. Recuperado de https://dialnet.unirioja.es/servlet/articulo?codigo=2776671

Programa de las Naciones Unidas para el Desarrollo. (2015). Objetivo de Desarrollo Sostenible. Recuperado de https://www.undp.org/content/undp/es/home/sustainable-development-goals.html

Pucheta, M. \& Bel, I. (2012). La diversidad de género en los comités de auditoría y la calidad de la información financiera. Revista contable, (3), 44-58. Recuperado de https://dialnet.unirioja.es/ejemplar/311630

Qi, B. \& Tian, G. (2012). The impact of audit committees' personal characteristics on earnings management: Evidence from China. Journal of Applied Business Research (JABR), 28(6), 13311344. DOI: https://doi.org/10.19030/jabr.v28i6.7347

Redhwan, A., Almahdi, A. \& Bakr, A. (2018). Are audit committee characteristics important to the internal audit budget in Malaysian firms?. Contaduría $y$ administración, 63(2), 947-969. DOI: https://dx.doi.org/10.22201/fca.24488410e.2018.1480

Robinson, D, \& Owens, L. (2009). Audit committee characteristics and auditor changes. Academy of Accounting and Financial Studies Journal, (13), 117-132 Recuperado de https://cutt.ly/7e5uvdo 
Rozas-Flores, A. (2014). El comité de auditoría. Quipukamayoc, 15(29), 33-42. DOI: https://doi.org/10.15381/quipu.v15i29.2067

Superintendencia Financiera de Colombia. (03 de octubre de 2014). Circular Básica Jurídica 029 de 2014. Recuperado de https://cutt.ly/ke5uzsu

Superintendencia Financiera de Colombia. (13 de julio de 1998). Circular 052 de 1998. Recuperado de https://cutt.ly/ne5ulaf

Superintendencia Financiera de Colombia. (29 de septiembre de 1999). Concepto No. 1999040689-2. Doctrinas y conceptos. Comité de auditoría. Recuperado de: https://cutt.ly/Qe5ujkj

Superintendencia Financiera de Colombia. (30 de septiembre de 2014). Circular 028 de 2014. Recuperado de https://cutt.ly/je5ux8x

Zarza, C., López, F. \& Reguera, N. (2018). The right person at the right time: Audit commitee members and the quality of financial information. Universia Bussines Review, (58), 18-35. Recuperado de: https://cutt.ly/ce5ubak

Zhang, Y., Zhou, J. \& Zhou, N. (2007). Auditt committee quality, auditor independence and internal control weakness. Journal of accounting and public policy. 26(3), 300-327. DOI: https://doi.org/10.1016/j.jaccpubpol.2007.03.001 